\title{
Production of Influenza Virus Proteins Using Recombinant Insect Cells
}

\author{
Takuya MAtsudA, Toshikazu TAniJima, Kyoko MASUMI-KoIZUMI, Tomohisa KATsudA, and Hideki YAMAJI* \\ Department of Chemical Science and Engineering, Graduate School of Engineering, Kobe University, 1-1 Rokkodai, Nada, Kobe \\ 657-8501, Japan
}

\begin{abstract}
Influenza vaccines have long been manufactured in embryonated chicken eggs. This method has some problems such as a long production period (about 6 months) and use of large amounts of infectious pathogens. Recently, the production of recombinant subunit vaccines using the baculovirus-insect cell system has been extensively investigated. In this system, viral immunodominant components can be produced more rapidly and in a larger scale than in the conventional egg-based process. However, continuous production is virtually impossible because infection of recombinant baculovirus results in the death of host insect cells. In the present study, we established stably transformed insect cells that secreted influenza virus-like particles (VLPs) consisting of hemagglutinin ( $\mathrm{HA})$, the major protective antigen of influenza $A$ virus, and matrix protein 1 (M1), another structural protein of the virus. Hemagglutination assay and transmission electron microscopy (TEM) suggested that HA produced by recombinant insect cells kept the hemagglutination activity and the morphology of the VLPs was similar to that of wild type influenza virus particles.
\end{abstract}

\section{Introduction}

Influenza is the infectious disease that annually causes approximately 500 million patients and hundreds of thousands of deaths worldwide. Influenza viruses are classified into types A, B, C, etc. and the influenza A and $B$ viruses routinely spread in people. Influenza $A$ virus is classified into subtypes like H1N1 and H7N9 according to the difference of two glycoproteins, hemagglutinin (HA) and neuraminidase (NA). Seasonal influenza is prevalent annually, and the epidemic strain defers from year to year. Therefore, the vaccine against seasonal influenza should be reconstituted every year. Pandemic influenza occurs by a new influenza A virus at intervals of decades and rapidly spreads worldwide, finally causing great damage to society. A pandemic influenza virus significantly differs from current and recently circulating human seasonal influenza A viruses. To confront the threat of pandemic influenza, it is necessary to develop the vaccine that contains novel components of the new virus in a short period. Hence, the manufacture of influenza vaccine requires rapidity and flexibility.

Traditionally, influenza vaccines have been manufactured in an egg-based process. In this process, influenza viruses are propagated in embryonated chicken eggs, and grown viruses are purified followed by inactivation and splitting. This process requires a long production period (about 6 months) including preparation of embryonated chicken eggs and processing of infectious pathogens (Gerdil, 2003). Therefore, there is concern that this process could not deal with the outbreak of pandemic influenza and increase in demand for seasonal influenza vaccines. As a rapid and flexible alternative to the eggbased process, some influenza vaccines have recently been manufactured in a mammalian cell culture-based process. However, in this process, vaccines are still produced from infectious pathogens that possibly cause safety issue.

Recently, the production of viral antigen proteins by using recombinant DNA technology has been developed as an alternative to a cell culture-based process. Subunit vaccines consisting of a single viral protein are produced in a recombinant protein production system, and they are safe because they do not contain viral genetic material (DNA and RNA). Several recombinant subunit vaccines have been approved in recent years. However, subunit vaccines often suffer from low immunogenicity probably caused by misfolded conformation of the target protein. Virus-like particles (VLPs) get attention as highly immunogenic subunit vaccines. VLPs are composed of viral surface proteins that spontaneously assemble into a particulate form, but they contain no viral genetic material. Therefore, unlike viruses, VLPs are non-infectious and non-replicating. VLPs elicit strong humoral and cellular immune responses like native virus particles, because of highly dense display of viral antigens in an authentic conformation (Yamaji, 2014). Therefore, VLPs are one of the most promising candidates of safe and effective vaccines.

\footnotetext{
Corresponding author: yamaji@kobe-u.ac.jp
} 
Among a variety of recombinant protein production systems, the baculovirus-insect cell system has been most extensively investigated for the production of VLPs. In this system, upon infection with a recombinant baculovirus carrying the gene of interest, insect cells produce considerable amounts of target proteins including virus antigen proteins. However, since the baculovirus infection results in the death of host insect cells, continuous production is virtually impossible in this system. Additionally, in the case of VLP production, progeny recombinant baculoviruses become contaminant and should be removed, though baculoviruses are not pathogenic for vertebrates (Vicente et al., 2011).

Stably transformed insect cells can be used as an alternative platform to the baculovirus-insect cell system without such problems (Yamaji, 2014). Insect cells can be cultured more easily and produce a larger amount of recombinant proteins than mammalian cells, which are often used for the production of therapeutic recombinant proteins. In addition, insect cells may be tolerant of the virulence of mammalian viruses. Accordingly, insect cells are suitable hosts for virus protein production (Kushnir et al., 2012). Furthermore, once stably transformed insect cells are established, target proteins can be produced only by culturing the cells. Recombinant insect cells may allow effective and continuous mass production of virus proteins including VLPs. In the present study, we investigated the production of influenza VLPs composed of HA, the major protective antigen of influenza A virus, and the matrix protein 1 (M1), another structural protein of the influenza virus, using stably transformed insect cells.

\section{Materials and Methods}

\subsection{Insect cells and culture medium}

The insect cell line Trichoplusia ni BTI-TN-5B1-4 (High Five; Thermo Fisher Scientific, Waltham, MA, USA) was used in the present study. Cell density was determined by microscopically counting the number of cells with a Countess II automated cell counter (Thermo Fisher Scientific), while cell viability was judged by trypan blue dye exclusion. The cells were maintained at $27^{\circ} \mathrm{C}$ in $\mathrm{T}$ -

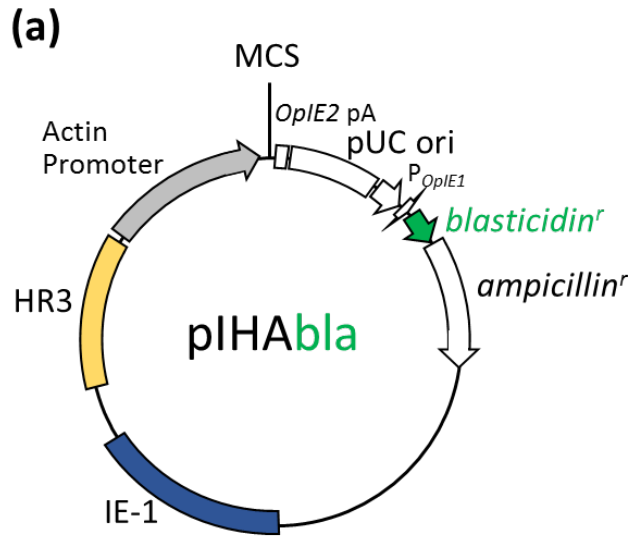

flasks in a non-humidified incubator. Express Five serumfree medium (Thermo Fisher Scientific) supplemented with $2.41 \mathrm{~g} / \mathrm{L}$ L-glutamine and $10 \mathrm{mg} / \mathrm{L}$ gentamicin was used.

\subsection{Plasmid construction}

In the present study, we used plasmid vectors, pIHAbla and pIHAneo (Figure 1) (Yamaji et al., 2008), which contained the Bombyx mori nucleopolyhedrovirus (BmNPV) IE-1 transactivator, the BmNPV HR3 enhancer, and the $B$. mori cytoplasmic actin promoter. The use of the IE-1 transactivator and the HR3 enhancer with the actin promoter has been shown to result in a significant high-level expression of a foreign gene (Douris et al., 2006). The pIHAbla and pIHAneo also contain either a blasticidin- or a neomycin-resistance gene for use as a selectable marker, respectively. The cDNA fragments encoding the influenza A virus (A/New Caledonia/20/1999 (H1N1)) envelope glycoprotein HA and the influenza A virus (A/Puerto Rico/8/1934 (H1N1)) M1 were PCR cloned into pIHAbla and pIHAneo, respectively (Figure 1).

\subsection{Transient expression}

High Five cells were inoculated into $35-\mathrm{mm}$ plastic culture dishes with $2 \mathrm{ml}$ of medium at a cell density of 2.0 $\times 10^{5}$ cells $/ \mathrm{cm}^{3}$. The cells were transfected with $1 \mu \mathrm{g}$ of plasmid using $3 \mu \mathrm{l}$ of FuGENE 6 transfection reagent (Promega, Madison, WI, USA). Three days after transfection, the culture supernatant was removed to evaluate the production of HA and M1. The culture supernatant was analyzed by sodium dodecyl sulfatepolyacrylamide gel electrophoresis (SDS-PAGE) and western blotting.

\subsection{Stable transformation}

High Five cells were inoculated into $35-\mathrm{mm}$ dishes with $2 \mathrm{ml}$ of medium at a cell density of $2.0 \times 10^{5}$ cells $/ \mathrm{cm}^{3} 1$ $\mathrm{h}$ before transfection. The cells were transfected with 1 $\mu \mathrm{g}$ of plasmid using $3 \mu \mathrm{l}$ of FuGENE 6 . Three days after transfection, the cells were removed from the dish and

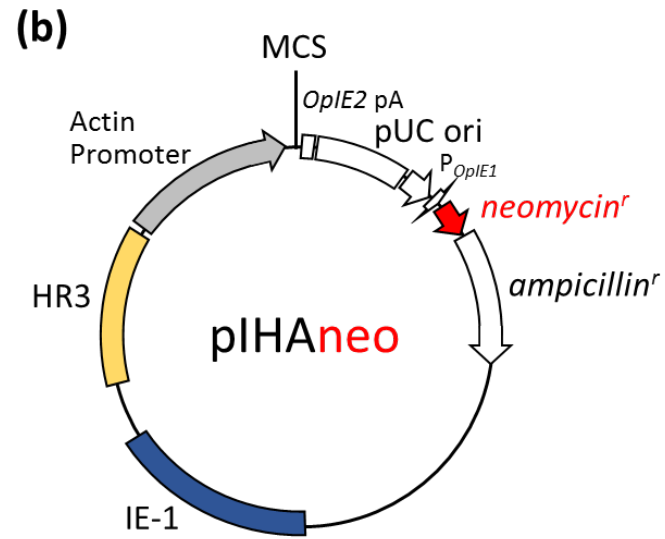

Figure 1. Expression plasmid vectors pIHAbla (a) and pIHAneo (b). MCS, maltiple cloning site 


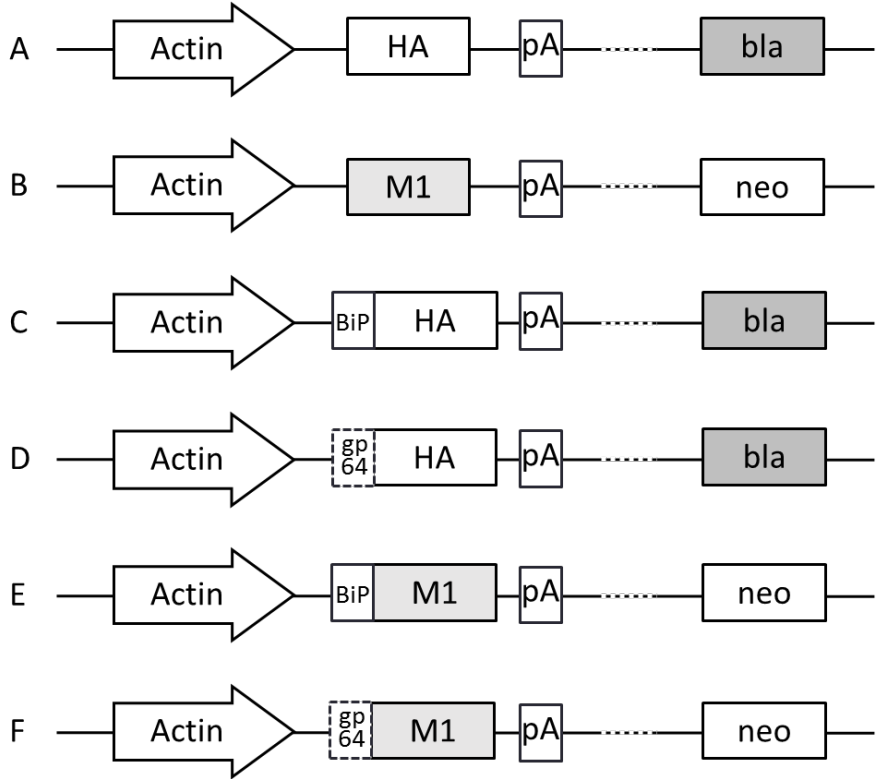

Figure 2. Schematic representation of plasmid vectors constructed. Actin, Bombyx mori cytoplasmic actin promotor; pA, OpIE2 polyadenylation sequence from Orgyia pseudotsugata nucleopolyhedrovirus (NPV); bla, blasticidin $^{\mathrm{r}}$; neo, neomycin ${ }^{\mathrm{r}}$; BiP, Drosophila BiP signal sequnce; gp64, Autographa californica NPV gp64 signal sequence

inoculated into a 100-mm plastic culture dish with $10 \mathrm{ml}$ of medium at a cell density of $0.8 \times 10^{5} \mathrm{cell} / \mathrm{cm}^{3}$. After 24-h incubation, blasticidin (Invivogen, San Diego, CA, USA) and G418 (Invivogen) were added at the concentrations of 22 and $280 \mu \mathrm{g} / \mathrm{ml}$, respectively, to select stable expression cells. The selective medium was replaced every 3 days until colonies of blasticidin- and neomycin-resistant cells were formed. Cells were isolated from each colony into a 96-well plate with $100 \mu \mathrm{l}$ of medium with blasticidin and G418. After the cells had grown to confluence, the cells were expanded gradually in a $35-\mathrm{mm}$ dish, a $25-\mathrm{cm}^{2}$ T-flask, and a $75-\mathrm{cm}^{2}$ T-flask. Stably transformed cells were frozen with Cell Reservoir One (Nacalai Tesque, Kyoto, Japan) at the concentration of $1.0 \times 10^{7}$ cells $/ \mathrm{cm}^{3}$.

\subsection{Production, concentration, and purification of VLPs}

Cells in the exponential growth phase were collected and suspended at a density of $2.0 \times 10^{5}$ cells $/ \mathrm{cm}^{3}$ in fresh medium. Fifteen, 50, and $200 \mathrm{ml}$ of cell suspension were transferred into 100-, 200-, and 500-ml screw-capped Erlenmeyer flasks, respectively. The cells in the flasks were cultivated at $27^{\circ} \mathrm{C}$ on a rotary shaker $(90 \mathrm{rpm})$.

After five days of culture, the supernatant was harvested and clarified by centrifugation for $5 \mathrm{~min}$ at 1000 $\times \mathrm{g}$. The supernatant was precipitated with $10 \%(\mathrm{w} / \mathrm{w})$ polyethylene glycol (PEG; molecular mass, approximately $6000 \mathrm{Da}$ ) and $1.9 \%(\mathrm{w} / \mathrm{w}) \mathrm{NaCl}$. After 2-h incubation, the pellets were collected by centrifugation for $20 \mathrm{~min}$ at $10,000 \mathrm{rpm}$ at $4^{\circ} \mathrm{C}$ using a $\mathrm{T} 15 \mathrm{~A} 39$ rotor (Koki Holdings, Tokyo, Japan). The pellets were then resuspended in phosphate buffered saline (PBS) and loaded on a 10 to $40 \%(\mathrm{w} / \mathrm{w})$ continuous sucrose density gradient in 2.2-ml tubes, and centrifuged at 55,000 rpm at $4^{\circ} \mathrm{C}$ for $1.5 \mathrm{~h}$ using an $\mathrm{S} 55 \mathrm{~S}$ swing rotor (Koki
Holdings). After centrifugation, fractions (150 $\mu \mathrm{l})$ were collected from the bottom of the tube and each fraction was analyzed by SDS-PAGE and western blotting. The fractions containing both HA and M1 were pooled and stored at $4^{\circ} \mathrm{C}$.

\subsection{Transmission electron microscopy (TEM)}

The culture supernatant precipitated with PEG and fractionated by sucrose density-gradient centrifugation was added onto an elastic carbon-coated copper grid (Okenshoji, Tokyo, Japan), and excessive solution was absorbed with filter paper. The sample was then stained with $2 \%$ phosphotungstic acid, and excessive stain solution was removed. The sample was observed using a JEM-2100F transmission electron microscope (JEOL, Tokyo, Japan) at $200 \mathrm{kV}$ at 100-200 k-fold magnification.

\subsection{Hemagglutination assay}

To evaluate the hemagglutination activity, a series of 2fold dilutions of the culture supernatant precipitated with PEG was prepared on a 96 -well plate. After $50 \mu \mathrm{l}$ of $1 \%$ chicken red blood cells (RBCs; Nippon Bio-test Laboratories, Saitama, Japan) was added, the plate was incubated at $25^{\circ} \mathrm{C}$ for $30 \mathrm{~min}$. Following incubation, the hemagglutination activity was detected visually in accordance with whether or not agglutination of RBCs was observed in the well.

\section{Results and Discussion}

\subsection{Establishment of stably transformed insect cells}


(a) M Sample
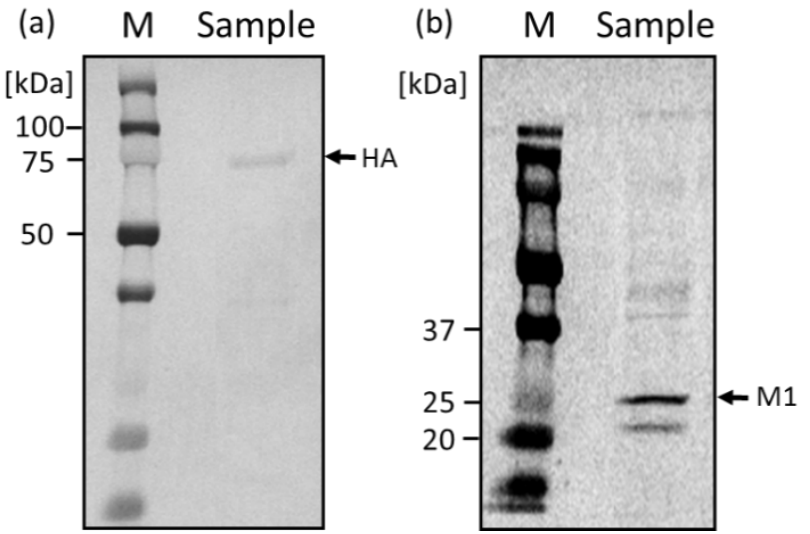

Figure 3. Western blot analysis of culture supernatant of High Five cells stably transformed with HA and M1 genes. Antibodies against HA (a) and M1 (b) were used as primary antibody. M, marker

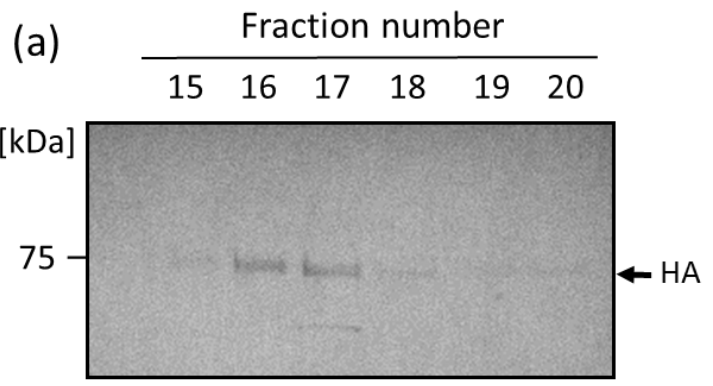

(b) Fraction number $\begin{array}{lllll}16 & 17 & 18 & 19 & 20\end{array}$

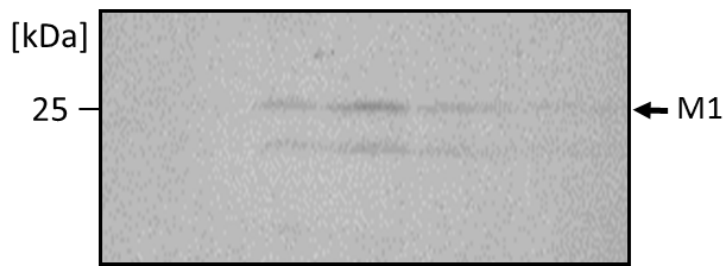

Figure 4. Western blot analysis of fractions obtained by precipitation with PEG and sucrose densitygradient sedimentation. Anti-HA (a) and antiM1(b) antibodies were used as primary antibody

(a)

(b)
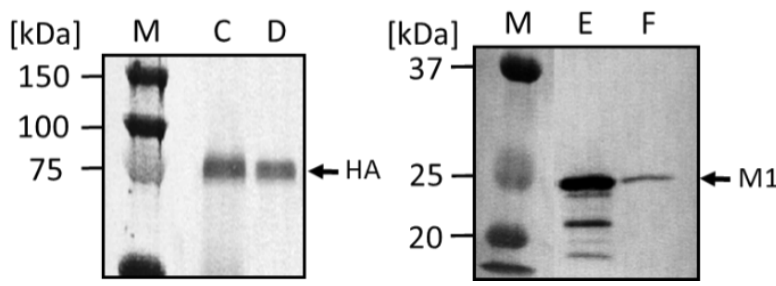

Figure 5. Western blot analysis of culture supernatant in transient expression of HA gene (a) and M1 gene (b) with different signal sequence. (a) Plamids $\mathrm{C}$ and $\mathrm{D}$ were used; (b) plasmids $\mathrm{E}$ and $\mathrm{F}$ were used
We constructed two plasmid vectors, pIHAbla-HA (Figure 2, A) and pIHAneo-M1 (Figure 2, B), which contained the influenza A virus $\mathrm{HA}$ and $\mathrm{M} 1$ gene, respectively, downstream of the BmNPV IE-1 transactivator, the BmNPV HR3 enhancer, and the $B$. mori actin promoter to establish stably transformed insect cells secreting influenza VLPs. After co-transfection with these two plasmids, High Five cells were cultured in the presence of blasticidin and G418, and cells resistant to the antibiotics were obtained. The culture supernatant of the resistant cells was analyzed by SDS-PAGE and western blotting. HA- and M1-specific bands were observed at an electronic mobility of approximately 75 and $25 \mathrm{kDa}$, respectively, which coincided with the molecular weight of HA and M1 (Figures 3 (a), (b)). This result suggests that established cells secreted HA and M1 molecules into the culture medium.

The culture supernatant of High Five cells stably transformed with HA and M1 genes was precipitated with PEG and fractionated by sucrose density-gradient sedimentation. HA and M1 molecules in each fraction were analyzed by western blotting (Figures 4 (a), (b)). HA- and M1-specific bands were detected in similar fractions (fractions 16-18). This indicates that HA and M1 molecules secreted from recombinant cells formed a complex in the culture supernatant.

\subsection{Comparison of signal sequence}

Next, we constructed four plasmid vectors (Figure 2, CF) in order to examine the effect of signal sequence on the expression level of HA and M1. Signal peptides target secreted proteins into the endoplasmic reticulum and may influence the production of secreted proteins (Yamaji and Konishi, 2013). The constructed plasmids contained the Drosophila immunoglobulin heavy chain binding protein (BiP) signal sequence (Yamaji et al., 2008) or the Autographa californica NPV (AcNPV) gp64 signal sequence (Jarvis et al., 1994) upstream of the HA or M1 gene. High Five cells were transfected with the resultant plasmids, and the culture supernatants obtained in transient expression were analyzed by western blotting. Figure 5 (a) shows that a higher yield of HA was obtained with the Drosophila BiP signal sequence compared with the AcNPV gp64 signal sequence. Similarly, the BiP signal sequence gave a higher yield of M1 than the gp64 signal sequence (Figure 5 (b)). Specific protein bands were detected at equivalent positions regardless of the signal sequence (Figures 5 (a), (b)) Therefore, the BiP signal sequence was used for the generation of stably transformed High Five cells expressing HA and M1 in subsequent investigations.

Novel stably transformed High Five cells were constructed by co-transfection using plasmids $\mathrm{C}$ and $\mathrm{E}$ and following incubation with blasticidin and G418. Among several cell clones resistant to the antibiotics, a highly productive clone of HA and M1 was successfully obtained (Figure 6, Clone 2). The novel stably transformed cells gave higher productivities of HA and M1 than the previously generated cells judging from the band intensity in western blotting (Figures 3 and 6), 
(a)

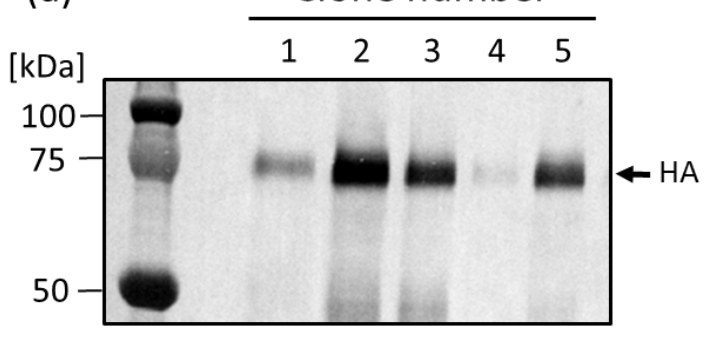

(b)

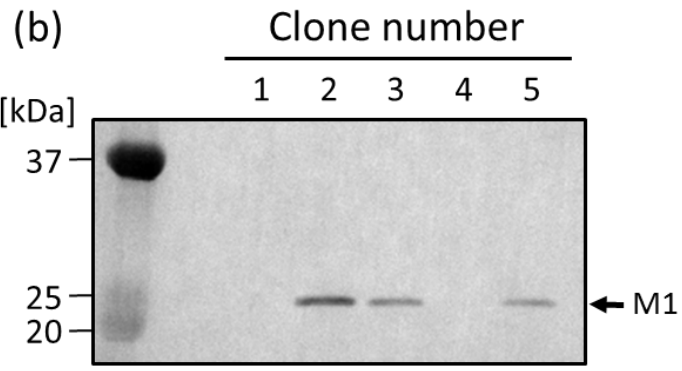

Figure 6. Comparison of several cell clones constructed using the Drosophila BiP signal sequence. Culture supernatants in static culture were analyzed by western blotting using anti-HA (a) and anti-M1 (b) antibodies
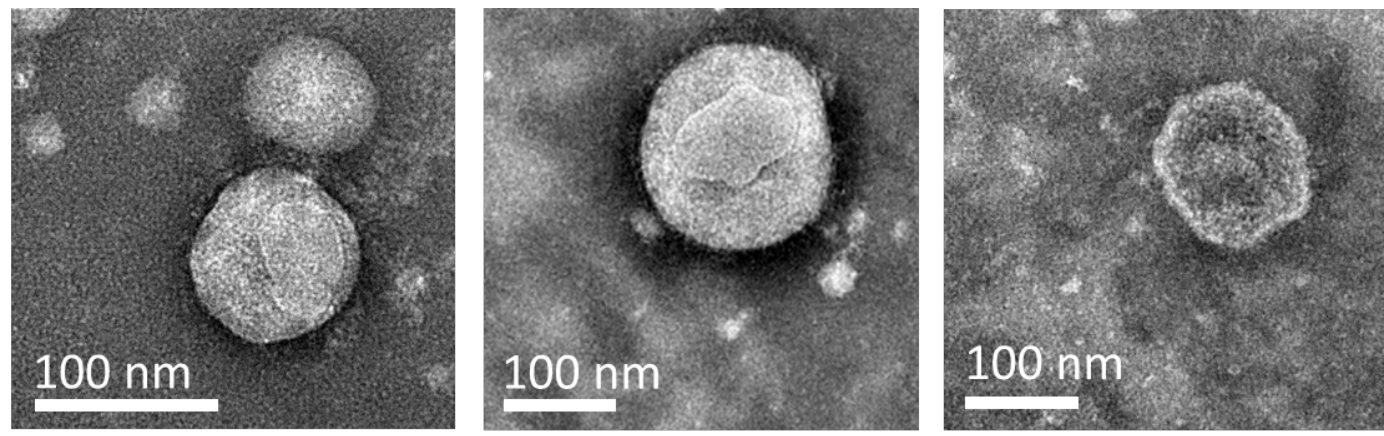

Figure 7. Transmission electron micrographs of culture supernatant precipitated with PEG and fractionated by sucrose density-gradient centrifugation

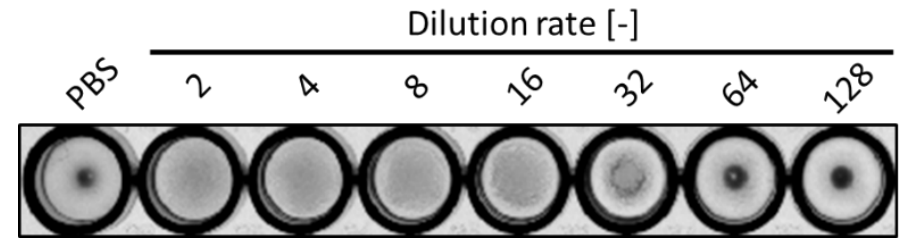

Figure 8. Hemagglutination assay of culture supernatant precipitated with PEG. Agglutination of chicken RBCs was observed at the dilution rates of 2-32.

suggesting that the BiP signal sequence would be useful for the generation of highly productive cells secreting HA and M1. By the method used in the present study, it was also demonstrated that only by culturing in the presence of two antibiotics, recombinant cells into which both the HA and M1 genes were integrated could be efficiently obtained.

\subsection{Characterization of influenza VLPs}

We further analyzed the culture supernatant of novel stably transformed cells by TEM and hemagglutination assay. Influenza viruses are enveloped viruses of which diameter is approximately 50-200 nm. HA molecules are embedded in the lipid bilayer envelope like spikes. Transmission electron micrographs of the culture supernatant precipitated with PEG and fractionated by sucrose density-gradient centrifugation are shown in Figure 7. The particles with lipid bilayer and spike structure were observed. The diameter of the particles was approximately 80-200 nm, which was consistent with those of influenza virus particles and influenza VLPs produced in other expression systems such as the baculovirus-insect cell system and a mammalian cell expression system (Schmeisser et al., 2012; Wei et al., 2011). Sucrose density-gradient sedimentation analysis (Figure 4) and transmission electron microscopy of culture supernatant (Figure 7) suggest that secreted HA and M1 molecules were produced in a particulate form. Although the data might be still preliminary, the present study demonstrates that recombinant High Five cells can produce and secrete influenza VLPs similar to the authentic virus particles.

Influenza HA molecules have the specific feature that they adsorb to RBCs. Based on this property, the hemagglutination assay and the hemagglutination inhibition assay were developed and have been used for the diagnosis of influenza viruses. Hemagglutination assay of the culture supernatant precipitated with PEG was conducted using 1\% chicken RBCs (Figure 8). Chicken RBCs were dispersed as a clump and agglutination of RBCs was observed in the wells with the dilution rates of 2-32 folds. This result indicates that the hemagglutination activity was present in the culture supernatant and HA molecules in the VLPs secreted by 
recombinant insect cells retained the hemagglutination activity. Further studies are in progress on quantitative analysis of HA and comparison of the productivity with other production systems.

\section{Conclusion}

In the present study, we investigated the production of influenza VLPs in recombinant insect cells. The cDNA fragments encoding HA and M1 of influenza A viruses were separately cloned into the plasmid vector pIHAbla and pIHAneo, respectively. After co-transfection with the prepared plasmids, High Five cells were incubated with blasticidin and G418, and cells resistant to the antibiotics were effectively obtained. The Drosophila BiP signal sequence was useful for the generation of highly productive cells secreting HA and M1. Sucrose densitygradient sedimentation analysis and transmission electron microscopy of the culture supernatant showed that secreted HA and M1 molecules were produced in a particulate form. Hemagglutination assay using chicken RBCs indicated that HA molecules in the VLPs retained the hemagglutination activity. Taken together, recombinant insect cells may offer a promising approach to the development and production of functional influenza VLPs.

\section{Acknowledgements}

This research was partially supported by the Japan Agency for Medical Research and Development (AMED) under Grant Number JP18ae0101054 and by a Grant-in-Aid for Scientific Research (B) (15H04195) from the Japan Society for the Promotion of Science.

\section{References}

Douris, V., L. Swevers, V. Labropoulou, E. Andronopoulou, Z. Georgoussi, and K. Iatrou; "Stably Transformed Insect Cell Lines: Tools for Expression of Secreted and Membrane-anchored Proteins and HighThroughput Screening Platforms for Drug and Insecticide Discovery," Adv. Virus Res., 68, 113-156 (2006)

Gerdil, C.; "The Annual Production Cycle for Influenza Vaccine," Vaccine, 21, 1776-1779 (2003)

Jarvis D. L. and A. Garcia, Jr., "Biosynthesis and Processing of the Autographa californica Nuclear Polyhedrosis Virus gp64 Protein," Virology, 205, 300313 (1994)

Kushnir, N., S. J. Streatfield, and V. Yusibov; "Virus-like Particles as a Highly Efficient Vaccine Platform: Diversity of Targets and Production Systems and Advances in Clinical Development," Vaccine, 31, 58-83 (2012)

Schmeisser, F., J. E. Adamo, B. Blumberg, R. Friedman, J. Muller, J. Soto, and J. P. Weir; "Production and Characterization of Mammalian Virus-like Particles from
Modified Vaccinia Virus Ankara Vectors Expressing Influenza H5N1 Hemagglutinin and Neuraminidase," Vaccine, 30, 3413-3422 (2012)

Vicente, T., A. Roldão, C. Peixoto, M. J. T. Carrondo, and P. M. Alves; "Large-Scale Production and Purification of VLP-based Vaccines," J. Invertebr. Pathol., 107, S42S48 (2011)

Wei H. J., W. Chang, S.-C. Lin, W.-C. Liu, D.-K. Chang, P. Chong, and S.-C. Wu; "Fabrication of Influenza Viruslike Particles Using M2 Fusion Proteins for Imaging Single Viruses and Designing Vaccines," Vaccine, 29, 7163-7172 (2011)

Yamaji, H.; "Suitability and Perspectives on Using Recombinant Insect Cells for the Production of Virus-like Particles," Appl. Microbiol. Biotechnol., 98, 1963-1970 (2014)

Yamaji H. and E. Konishi; "Production of Japanese Encephalitis Virus-like Particles in Insect Cells," Bioengineered, 4, 438-442 (2013)

Yamaji, H., T. Manabe, K. Watakabe, M. Muraoka, I. Fujii, and H. Fukuda; "Production of Functional Antibody Fab Fragment by Recombinant Insect Cells," Biochem. Eng. J., 41, 203-209 (2008) 